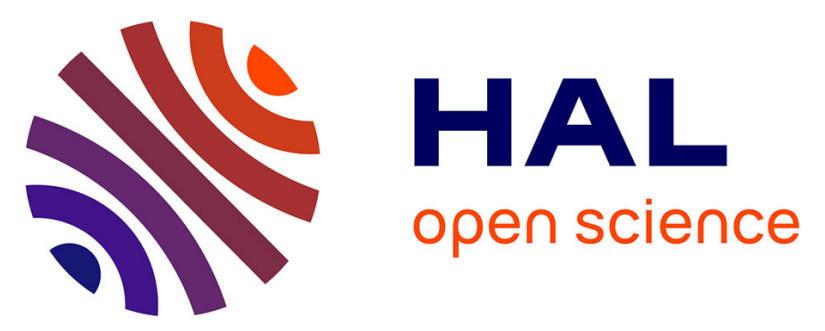

\title{
Systematic LFT Derivation of Uncertain Electrical Circuits for the Worst-Case Tolerance Analysis
}

Moises Ferber de Vieira Lessa, Anton Korniienko, Gérard Scorletti, Christian Vollaire, Florent Morel, Laurent Krähenbühl

\section{- To cite this version:}

Moises Ferber de Vieira Lessa, Anton Korniienko, Gérard Scorletti, Christian Vollaire, Florent Morel, et al.. Systematic LFT Derivation of Uncertain Electrical Circuits for the Worst-Case Tolerance Analysis. IEEE Transactions on Electromagnetic Compatibility, 2015, 57 (5), pp.937 - 946. 10.1109/TEMC.2015.2419455 . hal-01181163

\section{HAL Id: hal-01181163 https://hal.science/hal-01181163}

Submitted on 29 Jul 2015

HAL is a multi-disciplinary open access archive for the deposit and dissemination of scientific research documents, whether they are published or not. The documents may come from teaching and research institutions in France or abroad, or from public or private research centers.
L'archive ouverte pluridisciplinaire HAL, est destinée au dépôt et à la diffusion de documents scientifiques de niveau recherche, publiés ou non, émanant des établissements d'enseignement et de recherche français ou étrangers, des laboratoires publics ou privés. 


\title{
Systematic LFT Derivation of Uncertain Electrical Circuits for Worst-Case Tolerance
}

\author{
Analysis \\ Moises Ferber*†, Anton Korniienko*, Gérard Scorletti*, Christian Vollaire*, Florent Morel*, Laurent \\ Krähenbühl*, \\ ${ }^{*}$ University of Lyon, ECL \\ Ampere, CNRS UMR5005, 36 avenue Guy de Collongue, 69134 Ecully, France \\ Email: moisesferber@gmail.com \\ †Laboratorio de Computação Evolucionaria \\ Universidade Federal de Minas Gerais, Belo Horizonte, Brazil
}

\begin{abstract}
In line with the trend towards continuous miniaturization and price reduction, it is crucial to analyze the impact of uncertainties on the performance of electrical circuits. Performance is evaluated for the worst-case scenario and in the frequency domain, by computing upper and lower bounds. The purpose is not only to propose a method for the worst-case tolerance analysis but also to provide an efficient and a suitable tool for electrical engineers that can be easily applied to realistic electrical engineering problems. The proposed method is based on the robust analysis method (so called $\mu$-analysis) for which well-known and efficient algorithms exist. However in order to apply it, the problem under consideration has to be transformed in a standard minimal so called LFT representation. Its derivation is a difficult task even for control systems engineers. Our paper proposes a transparent and systematic LFT derivation procedure for users based only on their knowledge of electrical engineering. At the end of the paper, an industrial example is provided which reveals the benefits and the efficiency of the proposed approach and how it can be applied to any linear electrical circuit.
\end{abstract}

\section{Index Terms}

Linear Fractional Transformation, Robustness analysis of circuits, uncertainty, worst-case analysis, $\mu$-analysis, $\nu$-analysis.

\section{INTRODUCTION}

Nowadays, the semiconductor industry continuously scales toward nanometer technologies while constantly increasing complexity and deep silicon integration of electrical circuits. Both important technological dispersions and increase of interconnection parameter variations can bring a significant degradation of electrical circuit performance. 
In order to design a reliable system, it is thus required to take these parameter variations into account and to ensure the robust performance of the circuit besides the nominal one. The robust performance analysis is the procedure allowing to test whether the system performance remains to be acceptable in front of possible parameter variations or model uncertainties.

There can be underlined two existing approaches to achieve the mentioned goal: (i) probabilistic and (ii) worstcase analysis methodologies. The first approach allows to determine the probability of robust performance based on the given probability density functions (PDF) over parameters [1]-[5]. The worst-case approach in its turn does not consider any probabilistic aspects and the performance criteria is analyzed for the worst combination of the uncertain parameters [3], [6]-[10]. It ensures the robust performance for all possible combination of the uncertain parameters.

In some critical applications such as cardiac stimulation chips, nuclear power plant control, automatic driving panels of vehicles, etc., the ability to ensure the system performance in $100 \%$ of the cases is crucial. For these reasons, and due to the importance of such critical applications, the subject of this paper focuses on the worst-case tolerance analysis of electrical circuits. In this paper only linear electrical circuit models are addressed. Even though the linearity assumption could appear restrictive, in a large number of applications, the linear model of an electrical circuit precisely describing the behavior of the system around an operation point can still be derived. Furthermore, the worst-case robust performance analysis even for the linear systems but with general (possibly large) size and structure is actually a challenging NP-hard problem [11]. This means that the execution time of the algorithm is a Non-Polynomial function of the number of uncertainties considered.

There is a number of approaches to evaluate the uncertain system performance. In many applications related to circuit theory, such as, for example, filter or phase locked loop (PLL) analysis, the performance is evaluated in the frequency domain. In this case, the robust system performance is assessed by computing upper and/or lower bounds on the frequency responses of some performance transfer functions. These transfer functions are chosen such that they reflect the performance measure of the circuit. For the filter example, the performance transfer function is the filter attenuation measure between filter inputs and outputs.

The main purpose of the present paper is to propose a method for the worst-case tolerance analysis of any linear electrical circuit systems. The proposed method operates in frequency domain and should be efficient in terms of computational time (time grows as a polynomial function relative to the number of uncertainties) such that it is applicable for large-scale systems.

The importance of the problem under consideration is illustrated by the growing number of publications in this field [3], [6]-[10]. However, as it was pointed out before, the computational complexity and time is the main difficulty of the proposed solutions. In [6], [7] and [8], a methodology based on Interval Arithmetic (IA) is proposed. In order to simplify the computations, the authors enforce the assumption of monotonicity of the variable of interest with respect to the uncertain parameters. Transposing to the problem under consideration, it means that the frequency response magnitude of a filter can only grow if any of the uncertain parameters grows. This is a very strong assumption restricting the number of the electrical circuits that can be analyzed. In [7], the authors proposed 
an alternative solution based on interval partitioning which is again inefficient in terms of the computation time for large-scale systems. In [9], genetic algorithms (GA) and affine arithmetic (AA) are used to improve the results obtained from IA. However, the computational complexity is still high for systems of significant size and therefore its usage is somehow limited. An interesting solution to relax the monotonicity assumption is to encapsulate the bounds by outer and inner solutions as presented in [12]. However the authors in [12] consider only the steady states worst-case analysis and the dynamical aspects were put in perspective.

An interesting approach to address the problem considered in frequency domain is the $\mu$-analysis methodology originally proposed in the control system theory community [13]-[15]. This methodology proposes to deal with the computation complexity by relaxing complex analysis conditions. It leads to the convex Linear Matrix Inequality (LMI) optimization framework [16] for which efficient computation algorithms are available nowadays. The same idea of outer (inner) solutions as in [12] is used to perform this relaxation so that the performance analysis result is still ensured in $100 \%$ of the cases. However, in contrast to [12], the $\mu$-analysis can be applied for all frequencies which allows to include the dynamical system behavior and not only its steady states.

Nevertheless, though the $\mu$-analysis method is usually accepted in the control theory community for the worst-case performance analysis, very few results were published on its application by the electronic engineering community. Few exceptions can be found in [17], [18]. We believe that there are two main reasons that could explain this fact. The first reason is that the $\mu$-analysis method is a powerful theoretically based tool. It uses the control theory mathematical formalism which is not necessary the same as in the electrical engineering community and is rarely adapted to its practice. As a consequence, the interesting ideas are not understood and are not transmitted for practical use in electrical circuit systems. The second reason is that in order to apply the $\mu$-analysis, as it will be presented in this paper, a particular system transformation is necessary. This particular representation of the transformed system is called Linear Fractional Transformation (LFT) or $\Delta M$-representation. Once the LFT representation of the uncertain linear electrical circuit is derived, the application of robust worst-case performance analysis is a quite routine method based on the resolution of convex optimization problems involving LMI constraints.

For a typical automatic control application, it was proved that an LFT representation can be always obtained [19]. However, the choice of the LFT representation is not unique and the quality of the obtained result dramatically depends on this choice. In order to limit the computation burden and the numerical problems, an interesting choice is to find the so-called minimal LFT representation, that is, the LFT representation of the smallest dimension. This point is important since for non-academic applications, without a rigorous methodology, an LFT representation of an unnecessary high size is usually obtained. Such non-academic applications include the real industrial electrical circuits with an important number of components and uncertain parameters as well as several hierarchical levels.

Unfortunately, except for very particular classes of problems, the computation of the minimal LFT representation is a difficult and open problem [20]. Nevertheless, in the applications of the automatic control community, when the uncertain system is represented by a block diagram, a procedure was proposed to obtain from this block diagram, an LFT representation of the smallest size [21]. In the case when the block diagram representation is a minimal representation of the uncertain system, which is usually the case when the block diagram is rigorously constructed, 
the procedure leads to the minimal LFT representation. Nevertheless, in the electrical engineering community, the models of linear electrical circuits are not expressed as block diagrams but as electrical schematic. Even if it is theoretically possible, for real industrial electrical circuits, the transformation of an electrical schematic into a block diagram is a heavy, time-consuming task and thus it is not practically possible. It is then important to propose an efficient procedure to obtain an LFT representation directly from an electrical schematic.

In the few existing results on the application of the $\mu$-analysis to (uncertain) electrical circuit [17], [18], this crucial question was not addressed. The authors in [18] do not explain how to derive the LFT form, while in [17], the authors propose a manual and rather complex procedure based on matrix state space representations and block scheme interconnection that strongly depends on the example under consideration.

The main contribution of this paper is to propose a systematic procedure in order to obtain, for any linear electrical circuit, an LFT representation of reasonable dimension using the formalism of electrical schematic. Based on this representation, black-box procedures of the $\mu$-analysis approach allow to investigate the worst-case performance analysis of arbitrary linear electrical circuits. The major benefit is that an electrical engineer can directly apply the $\mu$-analysis to the worst-case tolerance analysis of arbitrary linear electrical circuits without special knowledge in control systems theory.

In section II, the problem formulation is given. In section III, based on the $\mu$-analysis theoretical result, the worst case upper bound problem is solved. Then in section IV, the systematic LFT representation derivation procedure of reasonable dimension is presented. In section $\mathrm{V}$, a general algorithm for worst case performance analysis is formulated and at the end of the paper, in section VI, an application numerical industrial example is presented. The article ends with a conclusion and further work discussions.

\section{NOTATIONS AND DEFINITIONS}

- Conjugate transpose of a matrix $F$ is denoted by $F^{*}$;

- Singular values $\sigma_{i}$ of a complex $n \times m$ matrix $F$ are defined as square roots of eigen values $\lambda_{i}$ of the matrix $F^{*} F$ if $n \geq m$ and $F F^{*}$ if $n<m$ i.e. $\sigma_{i}(F)=\sqrt{\lambda_{i}\left(F^{*} F\right)}$ for $n \geq m$ or $\sigma_{i}(F)=\sqrt{\lambda_{i}\left(F F^{*}\right)}$ for $n<m$ [22];

- $\sigma_{\max }(F)$ and $\sigma_{\min }(F)$ stand for the maximum and minimal singular value of the complex matrix $F$, respectively. For scalar matrix $F$, i.e. $n=m=1, \sigma_{\max }(F)=\sigma_{\min }(F)=|F|$;

- $\operatorname{dim}(w)$ denotes the dimension of the vector $w$;

- $I_{n}$ and $0_{n \times m}$ denote respectively an $n \times n$ identity and $n \times m$ zero matrices. The dimensions could be omitted if apparent from the context.

\section{Problem Statement}

A linear electrical circuit is a system of interconnected electrical components (see Table I). Each component is defined by a physical parameter. Once selected the structure of the linear electrical circuit, the physical parameters are chosen such that for certain input signals (currents or voltages) the Power Density Spectrum (PDS) of certain 
circuit signals (currents or voltage) satisfy certain lower and upper bounds. For this purpose, the linear electrical circuit is modeled by a transfer function $T_{w_{p} \rightarrow z_{p}}(s)$ such that

$$
z_{p}(s)=T_{w_{p} \rightarrow z_{p}}(s) w_{p}(s)
$$

where $z_{p}(s)$ denotes the Laplace transform of the system output, $w_{p}(s)$ the Laplace transform of the system input and $T_{w_{p} \rightarrow z_{p}}(s)$ is such that with $p=\left(p_{1}, \cdots, p_{N}\right)$ the vector of the $N$ parameters of the linear electrical circuit:

$$
T_{w_{p} \rightarrow z_{p}}(s)=\frac{\sum_{j=0}^{m} b_{j}(p) s^{j}}{\sum_{i=0}^{n} a_{i}(p) s^{i}} .
$$

The coefficients $a_{i}$ and $b_{j}$ of the transfer function $T_{w_{p} \rightarrow z_{p}}$ are in general rational functions of the $N$ parameters of the linear electrical circuit:

$$
a_{i}(p)=a_{i}\left(p_{1}, \cdots, p_{N}\right) \quad \text { and } \quad b_{j}(p)=b_{j}\left(p_{1}, \cdots, p_{N}\right) .
$$

It is a well-known fact that the lower and upper bounds on the PDS output signal can be recast as lower and upper bounds on the magnitude of the frequency response $T_{w_{p} \rightarrow z_{p}}(j \omega)$ [23]. Unfortunately, due to production or ageing etc. dispersions, the actual value of the parameters $p$ is different from the designed value $p^{0}$. A more adequate modeling is then to assume that each parameter $p_{k}$ belongs to an interval:

$$
p_{k} \in\left[p_{k}^{\min }, p_{k}^{\max }\right] .
$$

The center of the interval $p_{k}^{0}=\frac{p_{k}^{\max }+p_{k}^{\min }}{2}$ is referred to as the nominal value. If the actual value of $p_{k}$ is not known, the lower bound $p_{k}^{\min }$ and the upper bound $p_{k}^{\max }$ are a priori known. They are usually expressed as relative expression of the nominal value $p_{k}^{0}$, e.g.

$$
p_{k}=p_{k}^{0} \pm 50 \% \text {. }
$$

The question is then to evaluate for any frequency $\omega$ the range of variation of $|F(j \omega)|$ for any $p_{k} \in\left[p_{k}^{\min }, p_{k}^{\max }\right]$. This question can be decomposed into two different problems: the worst-case upper bound problem and the worstcase lower bound problem.

\section{WORST-CASE UPPER BOUND PROBLEM}

Given $T_{w_{p} \rightarrow z_{p}}, p_{k}^{\min }$ and $p_{k}^{\max }$, for a given frequency $\omega$ compute the smallest $\eta(\omega)$ such that for any $p_{k} \in$ $\left[p_{k}^{\min }, p_{k}^{\max }\right]$

$$
\left|T_{w_{p} \rightarrow z_{p}}(j \omega)\right| \leq \eta(\omega)
$$

Another interesting problem is the computation of the worst-case lower bound on the frequency response of the transfer function $T_{w_{p} \rightarrow z_{p}}$ : given $T_{w_{p} \rightarrow z_{p}}, p_{k}^{\min }$ and $p_{k}^{\max }$, for a given frequency $\omega$ compute the largest $\beta(\omega)$ such that for any $p_{k} \in\left[p_{k}^{\min }, p_{k}^{\max }\right]$

$$
\beta(\omega) \leq\left|T_{w_{p} \rightarrow z_{p}}(j \omega)\right|
$$


Nevertheless in the sequel, we focus on the WORST-CASE UPPER BOUND PROBLEM since the worst-case lower bound problem can be recast as an equivalent WORST-CASE UPPER BOUND PROBLEM with the upper bound $\widehat{\eta}(j \omega)$ defined as $\frac{1}{\beta(\omega)}$ and the transfer function $\widehat{T}_{w_{p} \rightarrow z_{p}}$ defined as $T_{w_{p} \rightarrow z_{p}}^{-1}$.

In the case when $z_{p}(s)$ and $w_{p}(s)$ are vectors (with $n_{z}=\operatorname{dim}\left(z_{p}\right)$ and $n_{w}=\operatorname{dim}\left(w_{p}\right)$ ) i.e. Multi-Input MultiOutput (MIMO) case, $T_{w_{p} \rightarrow z_{p}}(s)$ previously defined by (1) is now a matrix of transfer functions. The formulation of the WORST-CASE UPPER BOUND PROBLEM is extended as follows.

\section{MIMO WORST-CASE UPPER BOUND PROBLEM}

Given $T_{w_{p} \rightarrow z_{p}}, p_{k}^{\min }$ and $p_{k}^{\max }$, for a given frequency $\omega$ compute the smallest $\eta(\omega)$ such that for any $p_{k} \in$ $\left[p_{k}^{\min }, p_{k}^{\max }\right]$

$$
\sigma_{\max }\left(T_{w_{p} \rightarrow z_{p}}(j \omega)\right) \leq \eta(\omega)
$$

As in the single input, single output case, another interesting problem is the computation of the worst-case lower bound on the frequency response of the transfer function $T_{w_{p} \rightarrow z_{p}}$ : given $T_{w_{p} \rightarrow z_{p}}, p_{k}^{\min }$ and $p_{k}^{\max }$, for a given frequency $\omega$ compute the largest $\beta(\omega)$ such that for any $p_{k} \in\left[p_{k}^{\min }, p_{k}^{\max }\right]$

$$
\beta(\omega) \leq \sigma_{\min }\left(T_{w_{p} \rightarrow z_{p}}(j \omega)\right) .
$$

Nevertheless, in the sequel and for the same reasons as previously, we focus on the MIMO upper bound case only. In the next section, we reveal how the MIMO WORST-CASE UPPER BOUND PROBLEM is solved using robust control approach.

\section{SOLUTION TO WORST-CASE UPPER BOUND PROBLEM}

\section{A. Robust Performance Analysis}

According to (2), the uncertain parameters can be expressed as a sum of two parts: (i) the nominal part $p_{k}^{0}$ and (ii) the uncertain part $p_{k}^{0} p_{k}^{g} \delta_{k}$ :

$$
p_{k}=p_{k}^{0}\left(1+\delta_{k} p_{k}^{g}\right)
$$

where $p_{k}^{0}=\frac{1}{2}\left(p_{k}^{\max }+p_{k}^{\min }\right)$ denotes the nominal or center parameter value, $p_{k}^{g}=\frac{p_{k}^{\max }-p_{k}^{\min }}{p_{k}^{\max }+p_{k}^{\min }}$ the relative value of the uncertainty ${ }^{1}$ (for $50 \% \rightarrow p_{k}^{g}=0.5$ ) and $\delta_{k}$ is the corresponding normalized uncertainty $\left|\delta_{k}\right| \leq 1$.

The transfer function $T_{w_{p} \rightarrow z_{p}}$ defined by (1) can then be expressed as:

$$
T_{w_{p} \rightarrow z_{p}}(s)=\frac{\sum_{j=0}^{m} \widehat{b}_{j}\left(\delta_{1}, \cdots, \delta_{N}\right) s^{j}}{\sum_{i=0}^{n} \widehat{a}_{i}\left(\delta_{1}, \cdots, \delta_{N}\right) s^{i}} .
$$

\footnotetext{
${ }^{1}$ In (3), the relative uncertainty representation is introduced in order to limit the numerical problems during the further computations. However the absolute uncertainty form can be used as well.
} 
As the coefficients $\widehat{a}_{i}\left(\delta_{1}, \cdots, \delta_{N}\right)$ and $\widehat{b}_{j}\left(\delta_{1}, \cdots, \delta_{N}\right)$ are rational function of $\delta_{1}, \cdots, \delta_{N}, z_{p}(s)=T_{w_{p} \rightarrow z_{p}}(s) w_{p}(s)$ can always be transformed as [19]:

$$
\left[\begin{array}{c}
z(s) \\
z_{p}(s)
\end{array}\right]=M(s)\left[\begin{array}{c}
w(s) \\
w_{p}(s)
\end{array}\right] \text { and } w(s)=\Delta(s) z(s)
$$

where $\Delta$ is defined by

$$
\Delta=\left[\begin{array}{ccc}
\delta_{1} I_{n_{1}} & 0 & 0 \\
0 & \ddots & 0 \\
0 & 0 & \delta_{N} I_{n_{N}}
\end{array}\right]
$$

This representation, denoted ${ }^{2}$ to as $\Delta \star M$, is referred to as a Linear Fractional Transformation (LFT) representation of the linear electrical circuit. This representation can be interpreted as the feedback interconnection of the nominal part $M$ (usually designed to be stable) and the unknown part $\Delta$, see Fig.1.

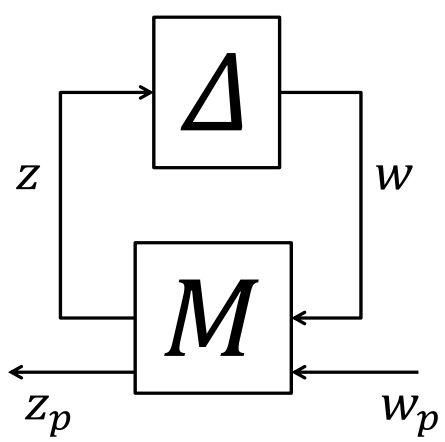

Fig. 1. LFT representation of the linear electrical circuit

Let us introduce the uncertainty set $\underline{\Delta}$ defined as:

$$
\Delta=\left\{\Delta \mid \Delta=\left[\begin{array}{ccc}
\delta_{1} I_{n_{1}} & 0 & 0 \\
0 & \ddots & 0 \\
0 & 0 & \delta_{N} I_{n_{N}}
\end{array}\right], \quad \begin{array}{l}
\forall k, \\
\delta_{k} \in \mathbb{R}
\end{array}\right\}
$$

The MIMO WORST-CASE UPPER BOUND PROBLEM can then be expressed as for a given $\omega$ : compute the smallest $\eta(\omega)$ such that for any $\Delta \in \underline{\Delta}$,

$$
\sigma_{\max }(\Delta \star M(j \omega)) \leq \eta(\omega)
$$

This problem referred to as the robust performance problem was largely investigated in the Automatic Control community. A solution is presented in the following theorem.

\footnotetext{
${ }^{2}$ The symbol $\star$ denotes the Redheffer star product, see e.g. [19].
} 
Theorem 1 (MIMO case, [24], Theorem 2.1): For stable nominal system $M(s)$, for all possible $\Delta \in \Delta$, for a given $\omega$

$$
\sigma_{\max }(\Delta \star M(j \omega))<\eta(\omega)
$$

if and only if there exists an hermitian matrix $\Phi(j \omega): \Phi^{*}(j \omega)=\Phi(j \omega)$ such that $t^{3}$

$$
\text { (i) } \forall \Delta \in \underline{\Delta},\left[\begin{array}{c}
I \\
\Delta
\end{array}\right]^{*}\left[\begin{array}{ll}
\Phi_{11} & \Phi_{12} \\
\Phi_{12}^{*} & \Phi_{22}
\end{array}\right]\left[\begin{array}{c}
I \\
\Delta
\end{array}\right] \geq 0
$$

and

$$
(i i)\left[\begin{array}{c}
M \\
I
\end{array}\right]^{*}\left[\begin{array}{cccc}
\Phi_{11} & 0 & \Phi_{12} & 0 \\
0 & I_{n_{z}} & 0 & 0 \\
\Phi_{21} & 0 & \Phi_{22} & 0 \\
0 & 0 & 0 & -\eta^{2} I_{n_{w}}
\end{array}\right]\left[\begin{array}{c}
M \\
I
\end{array}\right] \leq-\epsilon I
$$

with $\epsilon>0$

Based on this Theorem, the solution of WORST-CASE UPPER BOUND PROBLEM is readily obtained, see the following Corollary.

Corollary 1 (SISO case): For stable nominal system $M(s)$ and for all possible $\Delta \in \underline{\Delta}$, for a given $\omega$ :

$$
|\Delta \star M(j \omega)|<\eta(\omega)
$$

if and only if there exists an hermitian matrix $\Phi(j \omega): \Phi^{*}(j \omega)=\Phi(j \omega)$ such that (7) and (8) are satisfied with $n_{z}=n_{w}=1$.

\section{B. Computational Application of Theorem 1 and Corollary 1}

For a given frequency $\omega$, the application of Theorem 1 (respectively Corollary 1) allows to exactly find the upper bound $\eta(\omega)$ on the maximum singular value (frequency response magnitude) of $T_{w_{p} \rightarrow z_{p}}$ for MIMO (resp. SISO) case by computing an appropriate matrix $\Phi(j \omega)$ such that conditions (7) and (8) are satisfied.

However, it turns out that testing condition (7) is a difficult problem. Indeed, condition (7) has to be tested for all possible uncertainty $\Delta$ in the set $\underline{\Delta}$, i.e. an optimization problem involving an infinite number of constraints. In order to obtain an efficient computation, the optimization problem is modified by

1) parametrizing i.e. defining a set $\underline{\Phi}$ of matrices $\Phi(j \omega)$ for which the condition (7) is always satisfied;

2) testing the condition (8) for all $\Phi(j \omega)$ from this set i.e. for all $\Phi(j \omega) \in \underline{\Phi}$

In the case of a set $\underline{\Phi}$ which is affine or defined by LMI constraints, the constraint (8) of Theorem 1 defines a convex optimization problem involving a finite number of LMI constraints which is efficiently solved in polynomial time. Nevertheless, this optimization problem computes an upper bound $\bar{\eta}(\omega)$ on $\eta(\omega)$ instead of computing $\eta(\omega)$ itself. The consequence is a possible conservatism of the obtained result i.e. $\bar{\eta}(\omega)$ can be far away from $\eta(\omega)$. However, thanks to an appropriate choice of the parametrization set $\underline{\Phi}$, the conservatism can be significantly reduced.

\footnotetext{
${ }^{3}$ The dependency on $j \omega$ (and on $\omega$ ) is dropped for simplicity of notation.
} 
It is clear that the set $\underline{\Phi}$ depends on the uncertainty set $\underline{\Delta}$ and the better it describes the uncertainty throughout (7) the closer the relaxed upper bounds $\bar{\eta}(\omega)$ to the real ones $\eta(\omega)$ are. For our problem, a suitable set $\underline{\Phi}$ is defined ${ }^{4}$ by:

$$
\Phi=\left\{\Phi \mid \Phi=\left[\begin{array}{cccccc}
D_{1} & & 0 & G_{1} & & 0 \\
& \ddots & & & \ddots & \\
0 & & D_{N} & 0 & & G_{N} \\
-G_{1} & & 0 & D_{1} & & 0 \\
& \ddots & & & \ddots & \\
0 & & -G_{N} & 0 & & D_{N}
\end{array}\right]\right\}
$$

with $D_{i}$ and $G_{i}$ are complex, full, $n_{i} \times n_{i}$ hermitian and respectively skew hermitian frequency depending matrices i.e. $D^{*}=D$ and $G^{*}=-G$. It is so called $D, G$-scaling for the case of real uncertainties $\delta_{i}$ used in $\mu$-analysis [13], [14]. For this reasons, the use of the Theorem 1 with structure of $\Phi$ defined by (9) can be interpreted in terms of standard $\mu$-analysis.

Finally, the computation of the upper bounds (close to the real upper bounds) is thus achieved by solving for each frequency $\omega$ the following convex optimization problem under LMI constraints:

$$
\begin{aligned}
& \min _{\eta^{2}, \Phi} \eta^{2} \\
& \text { subject to (8) for } \Phi \in \underline{\Phi} \text { defined by (9) }
\end{aligned}
$$

The program solving the optimization problem (10) in Matlab environment, providing the Robust Toolbox is included in Matlab distribution, is available online on the website [25].

The MIMO WORST-CASE UPPER BOUND PROBLEM can be therefore efficiently solved in order to apply the worst-case performance analysis of the linear electrical circuit. However, this is possible only in the case where the LFT representation (5) corresponding to the electrical circuit is available. The next section presents how to obtain the LFT representation of reasonable size starting from the electrical circuit schematic.

\section{Systematic LFT DeRiVATION}

In this section, a method of systematic LFT derivation for general linear uncertain electrical circuits is presented. This is the crucial step to solve the worst-case tolerance analysis problem and, as discussed in the introduction, it is a difficult problem. In this paper, the following interesting idea is used to overcome this issue. When a designer is constructing the model of an electrical circuit with an arbitrary software ${ }^{5}$, interconnecting the library components together, creating and interconnecting then the subsystems in hierarchical way etc., he is actually building at the same time the LFT representation of the circuit with a minimal number of components. The only question is: how to extract this LFT representation (see $\Delta \star M$ in (5)) from the electric circuit model? For this purpose, once the

\footnotetext{
${ }^{4}$ The dependency on $j \omega$ (and on $\omega$ ) is dropped for simplicity.

${ }^{5}$ Example considered in this paper is the SimPowerSystems ${ }^{\mathrm{TM}}$ toolbox.
} 
electric circuit model is built, first the traditionally used component models (such as resistor, capacitor, inductor models etc.) are replaced by uncertain component models proposed in this section. The number and ordering of these uncertain components automatically defines the structure of the block $\Delta$. Then, the extraction procedure is performed in order to obtain the matrix transfer function $M(s)$.

In the following subsection, a library of elementary uncertain linear electric circuit components is proposed. Since these uncertain components are very similar to the standard ones, there is only the need of regular electrical engineer's knowledge to use (and build) the uncertain model. It is even possible to combine the regular and uncertain components in order to avoid time consuming computation in the case where some uncertainties can be neglected. Then, in next subsection, the procedure of LFT representation extraction is presented.

\section{A. Block Diagram of Uncertain Electrical Components}

The detailed description of uncertain components is given only for Resistor, Capacitor and Mutual Inductor while other components can be deduced in a similar fashion. A more complete table (or library) of uncertain components is then presented.

1) Resistor: The equivalent circuit of the normalized uncertain resistor is presented in the first line of the Table. I, where $R_{g}$ is the unit less normalization factor, $R_{0}$ is the resistor nominal value $(\Omega), \delta_{R}$ is a real number such that $\left|\delta_{R}\right|<1, z_{R}$ is the uncertain output, $w_{R}$ is the uncertain input, $i_{R}$ is the current that flows through the resistor and $v_{R}$ is the voltage drop across the resistor.

Note that the voltage seen by the terminals + and - of the circuit is the voltage drop on the resistor $R_{0}$ plus the voltage of the source in series with the resistor, which is given by $R_{0} i_{R} R_{g} \delta_{R}$. The total voltage drop between terminals + and - of the circuit is given by,

$$
\begin{aligned}
& v_{R}^{t o t}=R_{0} i_{R}+R_{0} i_{R} R_{g} \delta_{R}= \\
& \left(R_{0}+R_{0} R_{g} \delta_{R}\right) i_{R}=R_{0}\left(1+R_{g} \delta_{R}\right) i_{R}
\end{aligned}
$$

Thus, the equivalent resistance of the circuit corresponds exactly to the normalized relative form of uncertain parameter introduced in (3).

2) Capacitor: The equivalent circuit of the normalized uncertain capacitor is presented in the second line of Table I.

Note that the current that flows through the terminals + and - of the circuit is the current that flows through the capacitor $C_{0}$ plus the current that is injected by the source in parallel with the capacitor $C_{0}$, which is given by $C_{0} \frac{d v_{C}}{d t} C_{g} \delta_{C}$. The total current of the circuit is given by,

$$
\begin{aligned}
& i_{C}^{t o t}=C_{0} \frac{d v_{C}}{d t}+C_{0} \frac{d v_{C}}{d t} C_{g} \delta_{C}= \\
& \left(C_{0}+C_{0} C_{g} \delta_{C}\right) \frac{d v_{C}}{d t}=C_{0}\left(1+C_{g} \delta_{C}\right) \frac{d v_{C}}{d t}
\end{aligned}
$$

Thus the equivalent capacitance of the circuit exactly corresponds to the normalized relative form of uncertain parameter introduced in (3). 
3) Mutual Inductor: The equivalent circuit of the normalized uncertain mutual inductor is presented in the eighth line of Table I.

Note that the voltage $v_{1}$ seen by the terminals + and - in the left part of the circuit is the voltage drop on the inductor $L_{1}$, which is given by $L_{1} \frac{d i_{L_{1}}}{d t}$, plus the voltage drop on the Mutual Inductor $M_{0}$, which is given by $-M_{0} \frac{d i_{2}}{d t}$ and plus the voltage of the source in series with $M_{0}$, which is given by $-M_{0} \frac{d i_{2}}{d t} M_{g} \delta_{M}$. Thus, the total voltage $v_{1}$ in the left part of the circuit is given by,

$$
\begin{aligned}
v_{1}= & L_{1} \frac{d i_{1}}{d t}-M_{0} \frac{d i_{2}}{d t}-M_{0} \frac{d i_{2}}{d t} M_{g} \delta_{M} \\
& =L_{1} \frac{d i_{1}}{d t}-M_{0}\left(1+M_{g} \delta_{M}\right) \frac{d i_{2}}{d t}
\end{aligned}
$$

The procedure of obtaining $v_{2}$ is analogous. All other uncertain components from the proposed library are obtained in similar fashion and are presented in the Table I.

\section{B. LFT extraction}

In the last subsection, equivalent uncertain linear electrical circuits for uncertain linear components were developed. All these equivalent circuits have electrical terminals, for the usual electrical connections, but also two or four uncertain terminals, an input $w_{x}$ and output $z_{x}$, which should be connected to the uncertain block $\Delta$.

Thus, to automatically derive the matrix of transfer functions $M$ of any uncertain electrical circuit, one must follow the steps below:

1) connect the equivalent uncertain elements by their electrical terminals creating the desired circuit topology;

2) connect inputs and outputs to the uncertain terminals respecting the ordering (the $i^{\text {th }}$ element should have input $w_{i}$ and output $z_{i}$ ) according to the $\Delta$ structure, see Fig. 2;

3) connect the desired performance analysis input (voltage or current source) and output (voltage or current measurement), see Fig. 2;

4) compute a linear state-space model of $M$ and then the matrix transfer function $M(s)$. This last step can be performed, for example, in Matlab Simulink using the functions linmod $(\cdots)$ and ss $(\cdots)$.

In this section the systematic LFT derivation procedure was presented. Performing it together with the optimization problem (10) from section III allows to solve the MIMO wORST-CASE UPPER BOUND PROBLEM. The overall algorithm solving the worst-case tolerance analysis problem of linear electrical circuit i.e. upper and lower frequency bound computation, is given in next section.

\section{GENERAL ALGORITHM FOR WORST-CASE PERFORMANCE ANALYSIS}

As explained in the section II, the lower bound is obtained similarly to the upper bound by inverting the performance transfer function $T_{w_{p} \rightarrow z_{p}}=\Delta \star M$ and computing the upper bound of the inverted system $\left(T_{w_{p} \rightarrow z_{p}}\right)^{-1}=$ $(\Delta \star M)^{-1}$ :

$$
\left|T_{w_{p} \rightarrow z_{p}}(j \omega)\right|^{-1} \leq \beta^{-1}(\omega) \Leftrightarrow \beta(\omega) \leq\left|T_{w_{p} \rightarrow z_{p}}(j \omega)\right|
$$


TABLE I

UNCERTAIN COMPONENT LIBRARY

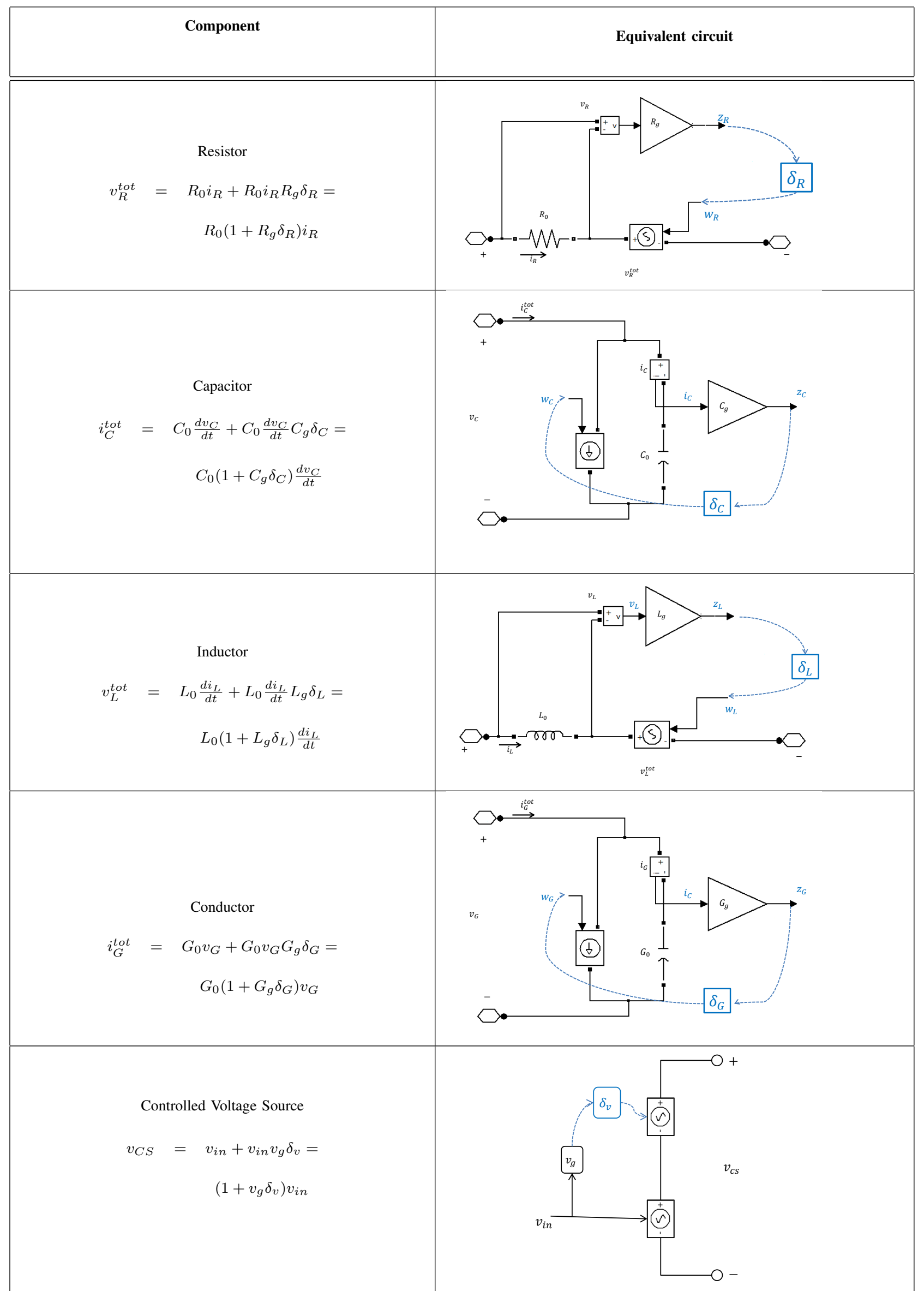


TABLE I

UNCERTAIN COMPONENT LIBRARY (CONTINUE)

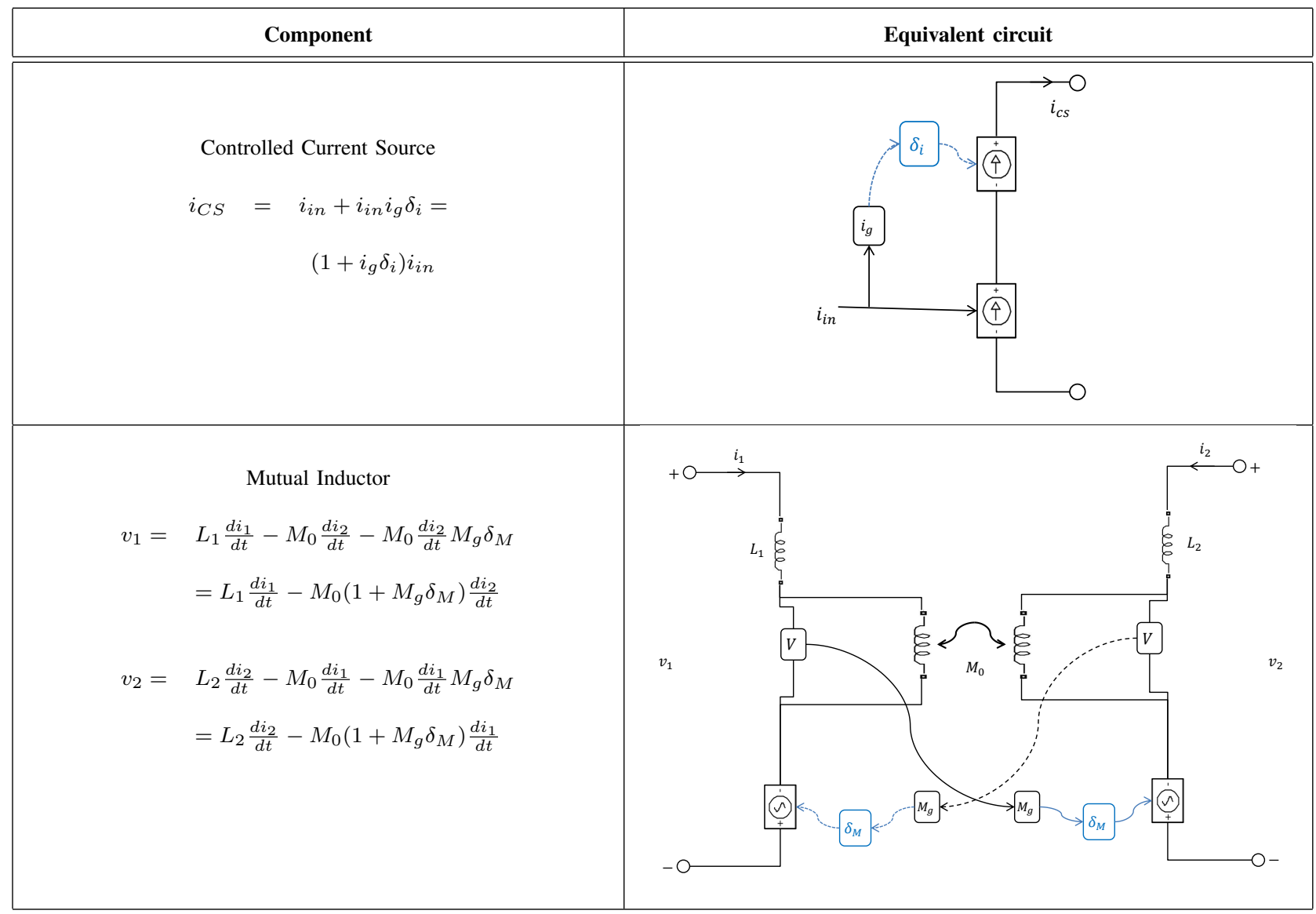

To do so, there is no need to recompute a new LFT representation and build a new electrical circuit. The corresponding inverse matrix transfer function $M_{i n v}$ can be computed based on the already computed direct matrix transfer function $M$ and thanks to the following LFT inversion relation [20]:

$$
M_{i n v}=\left[\begin{array}{cc}
M_{11}-M_{12} M_{22}^{-1} M_{21} & M_{12} M_{22}^{-1} \\
-M_{22}^{-1} M_{21} & M_{22}^{-1}
\end{array}\right]
$$

It should be noted that in the case of a non-invertible systems (zero static gain or non-causal inverse etc.), it is still possible to compute the corresponding inverse $M_{i n v}$ but frequency by frequency. In this case, for the frequencies with zero magnitude for nominal transfer function, the lower frequency bounds are zero as well and there is no need to compute the inverse matrix $M_{i n v}$.

The overall algorithm for the worst-case tolerance analysis of linear electrical circuits is now given. 


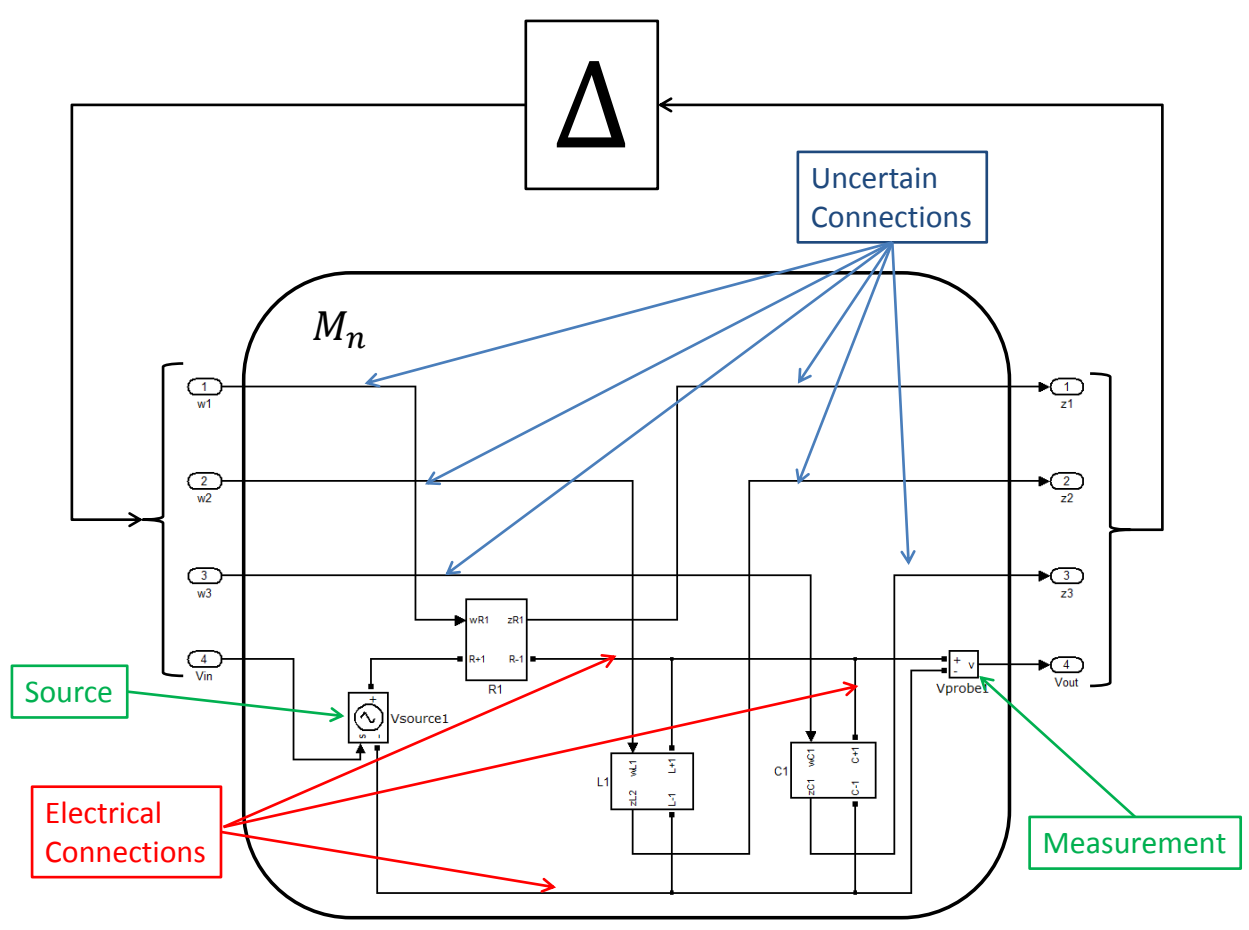

Fig. 2. Systematic extraction of LFT representation.

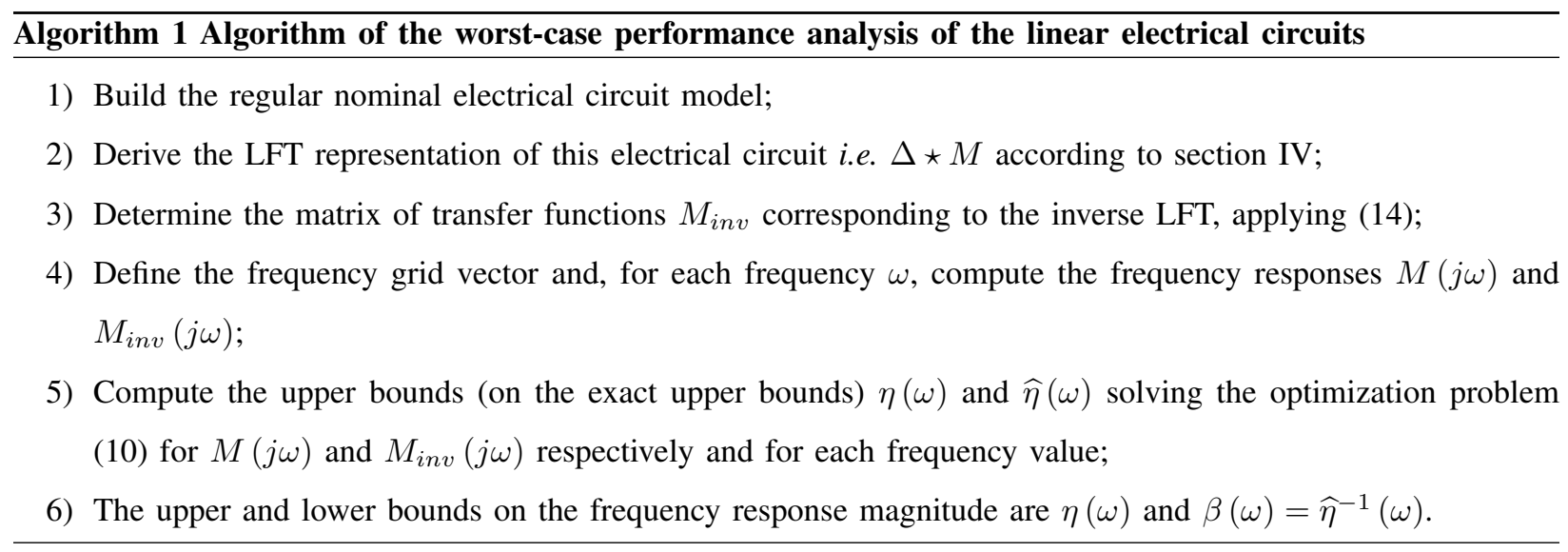

\section{Application}

The methodology presented in the previous section will be applied to an EMC filter. Its electrical schematic is shown in Fig. 3. It is a linear electrical circuit model with standard components R, C, L etc.. Since the filter is fabricated in industrial scale, technological dispersions causing parameter variations of these components are inevitable. In this example, there are 12 resistors, 9 inductors and 5 capacitors (including parasitic inductances and capacitances), which corresponds to 26 uncertain parameters. Computing the upper and lower bounds of the filter 
frequency response magnitude is therefore crucial in order to ensure an acceptable level of filtering performance.

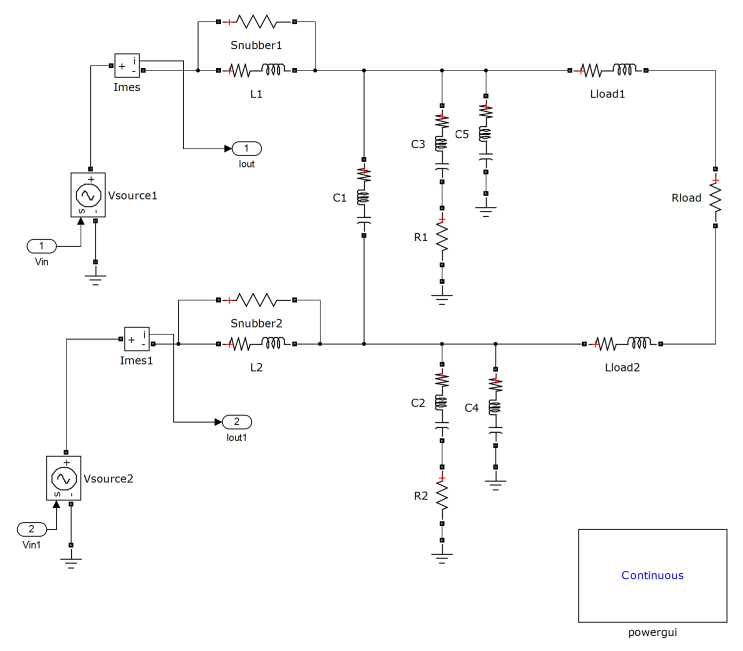

Fig. 3. Electrical schematic of EMC Filter.

Each electrical component is replaced by its uncertain block diagram and the matrices $M$ and $M_{i n v}$ of the filter are readily obtained from the diagram presented in Fig. 4 using linmod function of Matlab. As presented in Fig. 4, the structure of the model does not change with respect to the nominal model in Fig. 3, i.e. the same number of hierarchical levels, the same interconnection subsystems etc.. The only change concerns the additional uncertain inputs and outputs appearing for each uncertain component model. These inputs/outputs are propagated through every hierarchical level.

A frequency grid covering typical Conducted EMI standard's for this case was chosen. The computation of upper and lower bounds was then performed thanks to the function available [25]. The final results are presented in Fig. 5. In this figure, the full blue line represents the nominal filter frequency response magnitude. The red lines correspond to the computed upper and lower bounds, while dashed-dot green lines depict the frequency response magnitudes of the filter for the random chosen parameter combinations. For comparison reasons, the classical Monte-Carlo simulations were performed in order to compute the upper and lower frequency bounds as well.

As it can be seen in Fig. 5, no frequency response magnitude of randomly chosen parameter combinations (dashed-dot green lines) exceeds the bounds computed by our approach (red lines). Admittedly in contrast to our worst-case analysis approach, where the bounds are ensured, the Monte-Carlo bounds are only underestimation. Despite this, since Monte-Carlo simulations are a reference for robust performance analysis of electrical circuits (as well as for other domains), they should be discussed and compared with our approach. Furthermore, both the Monte-Carlo approach and our approach can be applied for any models of electrical circuit of arbitrary size and hierarchical structure.

First, the corresponding upper and lower bounds are close for both approaches. It is true that the Monte-Carlo bounds are tighter than those of the $\mu$-analysis. There are two reasons which explain the existence of such a 


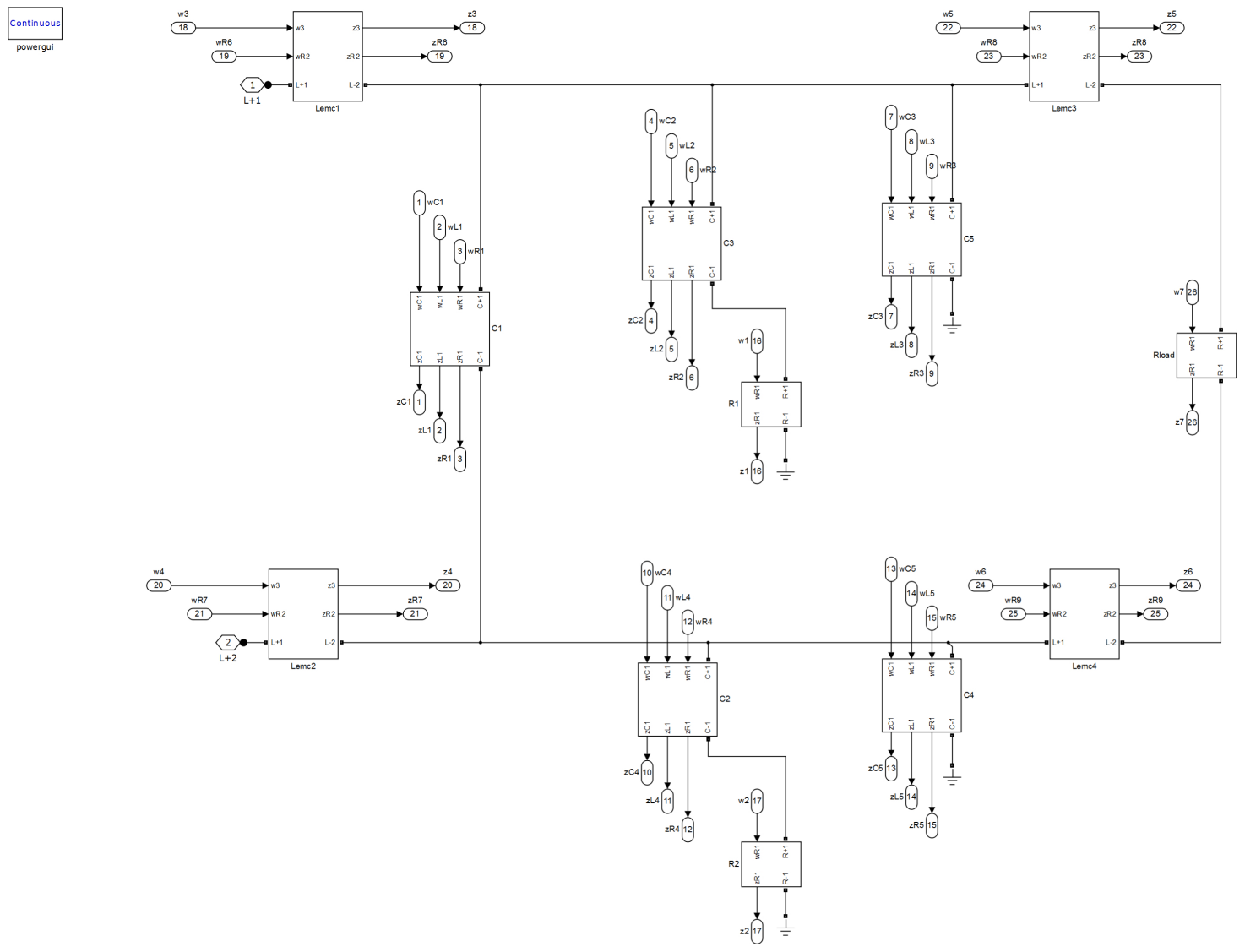

Fig. 4. Systematic LFT derivation of EMC Filter.

gap. The first is, as discussed previously, the possible conservatism of the result. This aspect can be improved by considering new types of parametrization sets $\underline{\Phi}$ for the optimization problem (10), see for example [24]. The second reason is, of course, the fact that the Monte-Carlo bounds are an estimation. This means that there are possible combinations of parameter values that may exceed the computed Monte-Carlo bounds. Ideally, if we could perform an infinite number of Monte-Carlo simulations, the new bounds will be closer to those of the $\mu$-analysis. This is confirmed by comparing Monte-Carlo bounds between $10^{4}$ and $10^{5}$ samples. The case of $10^{5}$ samples ensures a higher probability that the frequency response magnitude is inside of the computed bounds and it can be seen that these bounds approach the $\mu$-analysis upper and lower bounds.

Another comparison aspect is the computational efforts for two approaches. To obtain the bounds for $10^{4}$ samples, the computation time of Monte-Carlo approach is $t_{M C}^{4}=3$ hours and for the case of $10^{5}$ samples $t_{M C}^{5}=28$ hours. Whereas, in order to compute the bounds by $\mu$-analysis approach, ensured for all $100 \%$ cases, the computation time obtained is only $t_{\mu}=17$ minutes. The proposed method is almost 100 times quicker. These time comparisons were carried out on a Intel $17-2860 \mathrm{QM}$ Quad-Core $2.5 \mathrm{GHz}$. Thus, there is ample evidence of advantages of the proposed method when compared to the Monte-Carlo simulations. 


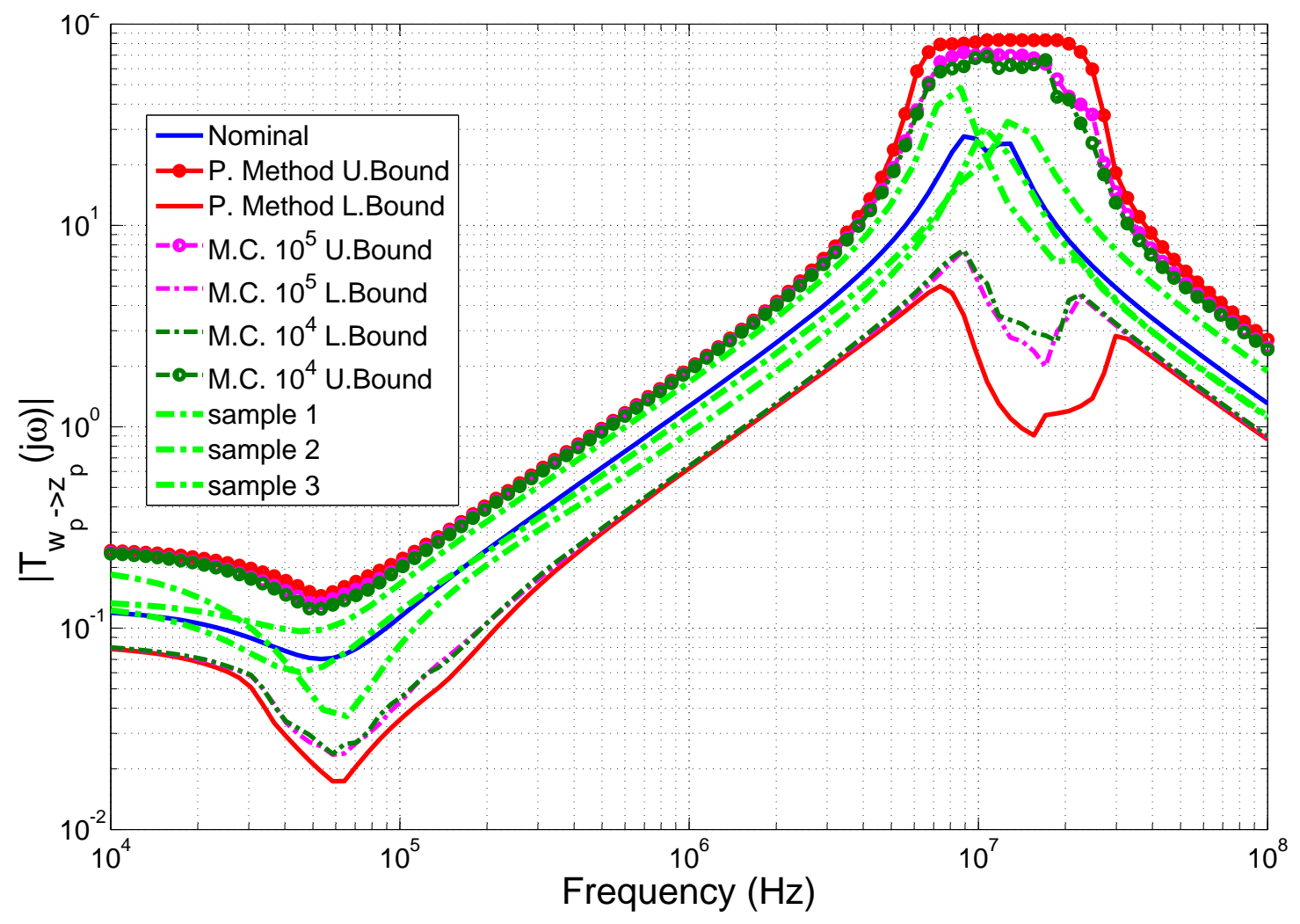

Fig. 5. Bounds computation results for EMC Filter.

\section{CONCLUSION}

In this paper, a frequency domain worst-case tolerance analysis method for uncertain linear electrical circuits was presented. The method is based on a robust control theory approach, the so called $\mu$-analysis. To apply the $\mu$-analysis, a particular LFT transformation problem must be considered first. The algorithm for systematic LFT derivation and worst-case tolerance analysis of linear electrical circuits was presented. To this purpose, a library of uncertain electrical components was created. The numerical example of EMC Filter proves the efficiency of the proposed approach compared to the classical Monte Carlo methodology.

Future works related to this paper will be an extension of the proposed approach to the case of the worst-case tolerance analysis along ranges of frequency, so that the appropriate choice of frequency grid can be easily performed. Other interesting perspectives are the systematic LFT reduction and the extension to the case of non-linear electrical circuits including switches.

\section{ACKNOWLEDGMENT}

This work was supported in part by Agence Nationale de la Recherche, programme VTT 2010, projet E-CEM. 


\section{REFERENCES}

[1] S. J. Julier, Jeffrey, and K. Uhlmann, "Unscented filtering and nonlinear estimation,” in Proceedings of the IEEE, 2004, pp. 401-422.

[2] L. de Menezes, A. Ajayi, C. Christopoulos, P. Sewell, and G. Borges, "Efficient computation of stochastic electromagnetic problems using unscented transforms," Science, Measurement Technology, IET, vol. 2, no. 2, pp. 88 -95, march 2008.

[3] H. Kettani and B. Barmish, "A new monte carlo circuit simulation paradigm with specific results for resistive networks," Circuits and Systems I: Regular Papers, IEEE Transactions on, vol. 53, no. 6, pp. 1289 -1299, june 2006.

[4] M. Ferber De Vieira Lessa, C. Vollaire, L. Krähenbühl, and A. Vasconcelos, Joao, "Adaptive unscented transform for uncertainty quantification in EMC large-scale systems," in Proc. of the 12th International Workshop on Optimization and Inverse Problems in Electromagnetism, Ghent, Belgium, Sep. 2012, p. CD.

[5] M. Ferber, C. Vollaire, L. Krahenbuhl, J.-L. Coulomb, and J. A. Vasconcelos, "Conducted EMI of DC-DC converters with parametric uncertainties," Electromagnetic Compatibility, IEEE Transactions on, vol. PP, no. 99, pp. 1 -8, 2013.

[6] S. Skelboe, "True worst-case analysis of linear electrical circuits by interval arithmetic," Circuits and Systems, IEEE Transactions on, vol. 26, no. 10, pp. 874-879, Oct 1979 .

[7] W. Tian, X.-T. Ling, and R. Liu, "Novel methods for circuit worst-case tolerance analysis," Circuits and Systems I: Fundamental Theory and Applications, IEEE Transactions on, vol. 43, no. 4, pp. 272-278, Apr 1996.

[8] M. Tian and C. J. R. Shi, "Worst case tolerance analysis of linear analog circuits using sensitivity bands," Circuits and Systems I: Fundamental Theory and Applications, IEEE Transactions on, vol. 47, no. 8, pp. 1138-1145, Aug 2000.

[9] N. Femia and G. Spagnuolo, "True worst-case circuit tolerance analysis using genetic algorithms and affine arithmetic," Circuits and Systems I: Fundamental Theory and Applications, IEEE Transactions on, vol. 47, no. 9, pp. 1285-1296, Sep 2000.

[10] J. Leyva-Ramos and J. Morales-Saldana, "Uncertainty models for switch-mode DC-DC converters," Circuits and Systems I: Fundamental Theory and Applications, IEEE Transactions on, vol. 47, no. 2, pp. 200-203, Feb 2000.

[11] R. D. Braatz, P. M. Young, J. C. Doyle, and M. Morari, "Computational complexity of $\mu$ calculation," IEEE Trans. Aut. Control, vol. AC-39, no. 5, pp. 1000-1002, May 1994.

[12] L. Kolev, "Worst-case tolerance analysis of linear DC and AC electric circuits," Circuits and Systems I: Fundamental Theory and Applications, IEEE Transactions on, vol. 49, no. 12, pp. 1693-1701, Dec 2002.

[13] J. Doyle, “Analysis of feedback systems with structured uncertainties," IEE Proc., vol. 129-D, no. 6, pp. 242-250, Nov. 1982.

[14] M. K. H. Fan, A. L. Tits, and J. C. Doyle, "Robustness in the presence of mixed parametric uncertainty and unmodeled dynamics," IEEE Trans. Aut. Control, vol. 36, no. 1, pp. 25-38, Jan 1991.

[15] S. Skogestad and I. Postlethwaite, Multivariable Feedback Control, Analysis and Design. John Wiley and Sons Chischester, 2005.

[16] Y. Nesterov, Introductory Lectures on Convex Optimization, ser. Applied optimization. Boston/Dordrecht/London: Kluxer Academic Publishers, 2004, no. 87.

[17] R. Tymerski, "Worst-case stability analysis of switching regulators using the structured singular value," Power Electronics, IEEE Transactions on, vol. 11, no. 5, pp. 723-730, Sep 1996.

[18] J. Wang, O. Hafiz, and J. Li, "A linear fractional transform (LFT) based model for interconnect parametric uncertainty”, in Design Automation Conference, 2004. Proceedings. 41st, July 2004, pp. 375-380.

[19] K. Zhou, J. Doyle, and K. Glover, Robust and Optimal Control. Prentice Hall, New Jersey, 1995.

[20] J. Doyle, A. Packard, and K. Zhou, "Review of LFTs, LMIs, and $\mu$," in Decision and Control, 1991., Proceedings of the 30th IEEE Conference on, Dec 1991, pp. 1227-1232 vol.2.

[21] S. Font, "Méthodologie pour prendre en compte la robustesse des systèmes asservis: optimisation $H_{\infty}$ et approche symbolique de la forme standard," Ph.D. dissertation, Université Paris XI Orsay, France, 1995.

[22] R. A. Horn and C. A. Johnson, Matrix Analysis. Cambridge University Press, 1985.

[23] R. Ozenbaugh and T. Pullen, EMI Filter Design - 3rd ed, ser. Electrical and computer engineering. CRC Press - Taylor \& Francis, 2012.

[24] G. Scorletti, X. Bombois, M. Barenthin, and V. Fromion, "Improved efficient analysis for systems with uncertain parameters," in IEEE Conf. Decision and Control, New Orleans, dec. 2007, pp. 5038-5043.

[25] [Online]. Available: https://sites.google.com/site/fastwctanalysis/ 